\title{
On the use of the Stokes number to explain frictional tidal dynamics and water column structure in shelf seas
}

\author{
A. J. Souza \\ National Oceanography Centre, Joseph Proudman Building, 6 Brownlow Street, Liverpool, L3 5DA, UK \\ Correspondence to: A. J. Souza (ajso@noc.ac.uk) \\ Received: 15 November 2012 - Published in Ocean Sci. Discuss.: 10 December 2012 \\ Revised: 27 February 2013 - Accepted: 12 March 2013 - Published: 2 April 2013
}

\begin{abstract}
In recent years coastal oceanographers have suggested using the "Strouhal" number or its inverse, the "Stokes" number, to describe the effect of bottom boundary layer turbulence on the vertical structure of both density and currents. These are defined as the ratios of the frictional depth $(\delta)$ to the water column depth $(h)$ or vice versa. Although many researchers have mentioned that the effects of the earth's rotation should be important, they have tended to omit it. Rotation may have an important influence on tidal currents, as the frictional depth from a fully cyclonic to a fully anticyclonic tidal ellipse can vary by up to an order of magnitude at mid latitudes. The Stokes number might appear smaller for cyclonic current ellipses (larger for anticyclonic) than it is without rotation, resulting in frictional effects being underestimated (overestimated). Here, a way to calculate a Stokes number is proposed, in which the effect of the earth's rotation is taken into account. The standard Stokes and the rotational Stokes numbers are used as predictors for the position of the tidal mixing fronts in the Irish Sea. Results show that use of the rotational number improves the predictions of fronts in shallow cyclonic areas of the eastern Irish Sea. This suggests that the effect of rotation on the water column structure will be more important in shallow shelf seas and estuaries with strong rotational currents.
\end{abstract}

\section{Introduction}

Stokes (1851) studied the flow over an oscillating plate (analogous to oscillatory flow over the bottom) and defined the depth of frictional influence denoted by the parameter $\delta$. The ratio of $\delta$ to the total water column depth $(h)$ is known as the Stokes number:

Stk $=\frac{\delta}{h}$.

This is equivalent to the ratio of friction to local accelerations in the momentum balance. If we define the oscillatory boundary layer thickness following Lamb (1932),

$\delta=\frac{C_{1} U_{*}}{\omega}$,

where $\omega$ is the oscillatory frequency, e.g. the $\mathrm{M}_{2}$ semi-diurnal frequency, and $U_{*}$ is the frictional velocity $\left(U_{*}=C_{\mathrm{d}}{ }^{1 / 2} U\right)$, where $U$ is the $\mathrm{M}_{2}$ velocity amplitude, $C_{\mathrm{d}}$ is the quadratic drag coefficient, and $c_{1}$ is a proportionality constant. The Stokes number can be expressed as follows:

$\mathrm{Stk}=\frac{c_{1} U_{*}}{\omega h}=\frac{\delta}{h}$.

The Strouhal number was originally defined by Strouhal (1878) while experimenting with wires experiencing vortex shedding and singing in the wind. This number is now used mainly to explain vortex shedding. The Strouhal number is defined as follows:

$\operatorname{Str}=\frac{\omega L}{U}$,

where $\omega$ is the frequency of the vortex shedding, $L$ is a characteristic length and $U$ is the velocity of the flow.

In recent years the Strouhal number has commonly been used to assess the effect of friction in oscillatory flows and has been defined as following:

$\mathrm{Str}=\frac{\omega h}{c_{1} U_{*}}=\frac{h}{\delta}=\frac{1}{\mathrm{Stk}}$, 
as in Baumert and Radach (1992) and Souza et al. (2013), or simply

$\operatorname{Str}=\frac{\omega h}{U}$,

e.g. as in Prandle (1982) or Burchard and Hetland (2011). Although this analysis is dimensionally correct, the use of the Strouhal number is dynamically incorrect as it is the ratio of local to advective accelerations.

The aim of this paper is three-fold: (1) to establish the use of the Stokes number as the correct number to be used when considering the effects of friction over inertia, (2) to highlight the effect of the earth's rotation on frictional tidal processes, and (3) To use the rotational Stokes number as a predictor of tidal mixing fronts as a test of the influence Earth's rotation.

\section{Use of the Stokes number to describe tidal dynamics in estuaries and Shelf Seas}

Stokes (1851) explained this problem, in what has become known as the Stokes "second problem" in fluid mechanics; it has been published in several textbooks and it is applicable to any oscillatory case (i.e. waves and tides).

The original problem was the flow due to an oscillating plate, in which the governing equation is

$$
\frac{\partial u}{\partial t}=N_{z} \frac{\partial^{2} u}{\partial z^{2}}
$$

with boundary condition $u(0, t)=U \cos \omega t$, and had a solution as follows:

$u=U e^{-z\left(\frac{\omega}{2 N_{z}}\right)^{1 / 2}} \cos \left(\omega t-z\left(\frac{\omega}{2 N_{z}}\right)^{\frac{1}{2}}\right)$,

where $N_{z}$ is the eddy viscosity, and $U$ and $\omega$ are the amplitude and frequency of the oscillating motion.

The height of frictional influence or Stokes depth is given by following equation:

$\delta \sim\left(\frac{2 N_{z}}{\omega}\right)^{1 / 2} \sim \frac{c_{1} U_{*}}{\omega}$

in the case of oscillatory flow forced by a horizontal pressure gradient due to the surface slope, as in the case of waves and tides. The equations of motions are

$\frac{\partial u}{\partial t}=N_{z} \frac{\partial^{2} u}{\partial z^{2}}-g \frac{\partial \eta}{\partial \chi}$

where $\eta$ is the surface elevation and $g$ is gravity. With the pressure gradient given by the following equation,

$-g \frac{\partial \eta}{\partial x}=\mathrm{A} \cos (\omega t)$ and boundary conditions $u=0$ at $z=0$, and $\partial u / \partial z=0$ at $z=h$, we have the following solution:

$u=\frac{A}{\omega}\left(\sin \omega t-e^{\frac{-z}{\delta}} \sin \left(\omega t-\frac{z}{\delta}\right)\right)$.

We can assume that the velocity far away from the boundary in the free stream will be given by

$u_{\infty}=\frac{A}{\omega} \sin \omega t$.

If $u$ is normalised by $A / \omega$ and we substitute $\zeta=z / h$, the solution becomes as follows:

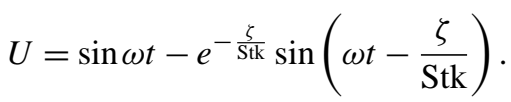

This is a similar result to Ianniello (1977) and demonstrates how the Stokes number determines the profiles and residual currents in well-mixed tidal flow in closed estuaries.

Figure 1 shows the normalised velocity profile $(U)$ for different Stokes numbers. As the Stokes number increases, the height of penetration increases, so at small Stoke numbers the bottom boundary layer $(\delta)$ is small, as would be the case for wind waves or tides in the deep ocean $(\mathrm{Stk}=0.01)$; as the Stoke number approaches unity, the boundary layer will cover the entire water column. Figure 2 shows the velocity distribution at different Stokes numbers spanning four orders of magnitude from 0.01 to 100 . The top plot shows normalised amplitude, and the bottom plot the phase lag.

Prandle (1982) and Prandle et al. (2011) found an analogous solution; they show how the amplitude and phase of tidal currents vary as a function of what they called the "Strouhal" number, but should really be the Stokes number, similar to Fig. 2. They even defined a solution for the rotational case, defining the clockwise and anticlockwise components of the Strouhal number, and suggest that the behaviour of each rotational component of velocity will behave following their individual Strouhal number.

Baumert and Radach (1992) identified the Strouhal number, as a characteristic parameter for the mixing associated with tidal flow. By demonstrating that, apart from bottom and surface roughness lengths, Str is the only parameter defining the dynamics of the well-mixed irrotational pressure gradient driven tidal flow, they could show how the relative time lag of turbulent parameters with respect to the bed stress increases with Str, in a similar way to Simpson et al. (2000) and Souza et al. (2004). Burchard (2009), Burchard and Hetland (2010) and Souza et al. (2013) have used this idea to define the behaviour of tidal and oscillatory flows in shelf seas using turbulent closure models. It needs to be mentioned that Prandle (1982), Baumert and Radach (1992), Burchard (2009) and Burchard and Hetland (2010) have defined the Strouhal wrongly as its inverse.

Winant (2007) used the Stokes number (his $\delta$ parameter) to define the tidal behaviour on elongated rotating estuaries, 


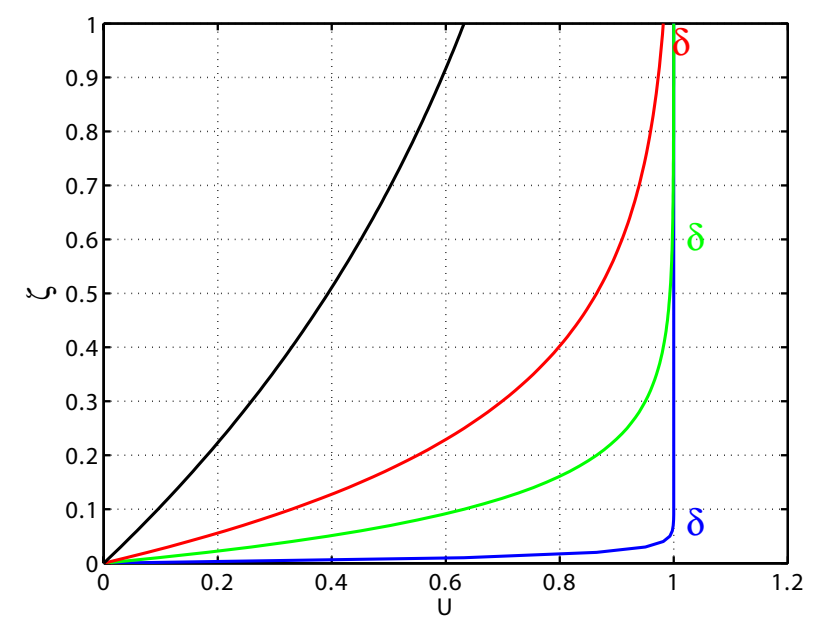

Fig. 1. Schematic showing the amplitude velocity profile for an oscillatory current with different Stokes number; blue, green red and black have Stokes numbers of $0.01,0.1,0.25$ and 1 , respectively. The elevation has been normalised by the total water depth and the velocity by the free stream velocity. The position of the height of frictional influence is depicted by $\delta$.

while Winant (2008) and Huijts et al. (2011) used it to describe the transverse structure of residual flows in estuaries. Although both authors suggest that the earth's rotation is important, neither of them took it into account when defining the Stokes number, i.e. the frictional depth.

\section{The effect of the earth's rotation on the Stokes number}

The Stokes number accounts for the relative dynamical importance between the frictional and the local accelerations. Due to the fact that any value larger than one would mean that the frictional layer is greater than the water depth, in practical terms it will be considered to cover the full water column.

To explain the elliptical behaviour of tidal currents, it is better to decompose the velocity vector into rotational components (Thorade, 1928; Godin, 1972; Prandle, 1982): one rotating cyclonically $\left(R_{+}\right.$anticlockwise in the Northern Hemisphere) and another rotating anticyclonically $\left(R_{-}\right.$ clockwise).

Following Souza and Simpson (1996), the tidal ellipses' semi-major $\left(U_{M}\right)$, semi-minor $\left(U_{m}\right)$ and ellipticity $(\varepsilon)$ are given by

$U_{M}=R_{+}+R_{-}$,

$U_{m}=R_{+}-R_{-}$,

and
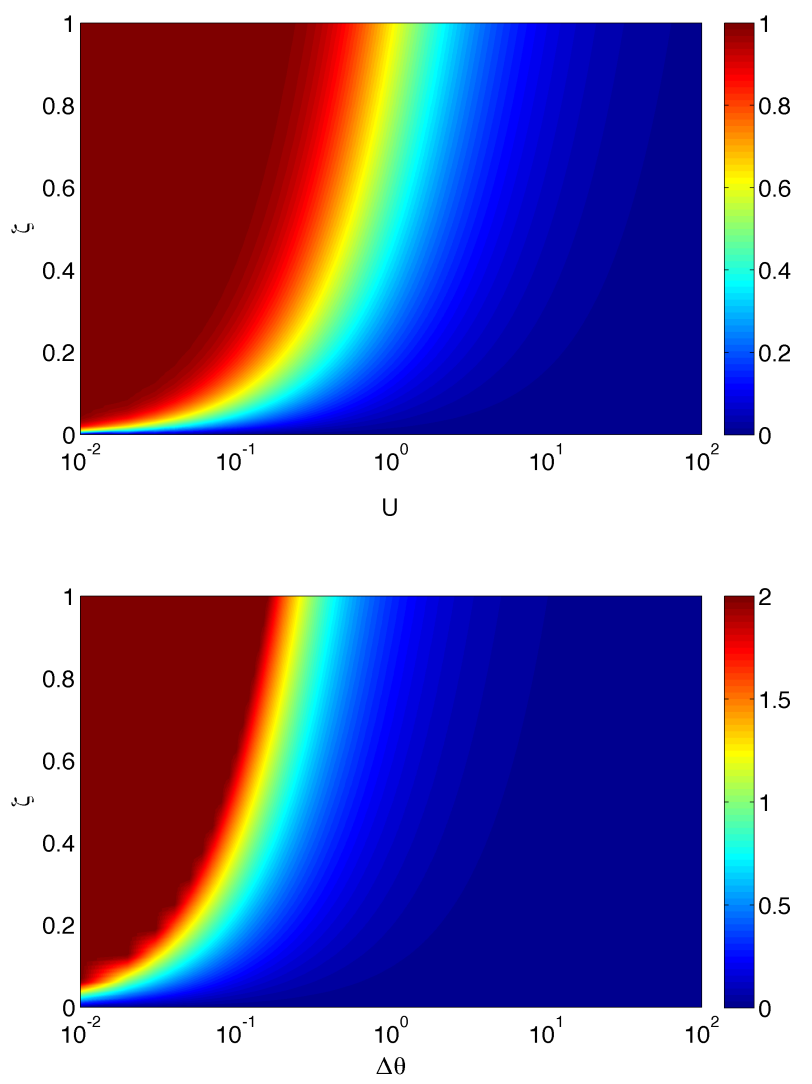

Fig. 2. Tidal current profile as a function of the Stokes number. Distribution of normalised amplitude velocities (top), and phase difference in pi radians (bottom).

$\varepsilon=\frac{U_{m}}{U_{M}}$.

Each of these rotational velocity components has a characteristic boundary layer thickness (Fig. 3) as explained in Souza and Simpson (1996). By analogy with Eq. (3) the boundary layer thickness, as given by Soulsby (1983), is

$\delta_{+}=\frac{c_{2} U_{*}}{(\omega+f)}$

for the cyclonic and

$\delta_{-}=\frac{c_{2} U_{*}}{(\omega-f)}$

for the anticyclonic components, where $f$ is the Coriolis frequency. In mid-latitudes $f$ and $\omega$ are close so that $\delta_{-} \gg \delta_{+}$, hence the anticyclonic component will cover more of the water column (Fig. 3). In the case of mid-latitudes, the ratio of the counter-rotational boundary layers is

$\frac{\delta_{-}}{\delta_{+}}=\frac{\omega-f}{\omega+f} \sim 10$. 
Table 1. Frictional layer depth and stokes number for a $1 \mathrm{~ms}^{-1}$ tidal current with different ellipticities at $54^{\circ} \mathrm{N}$ and $30 \mathrm{~m}$ water column depth.

\begin{tabular}{l|rrrrrrr}
\hline$\varepsilon$ & -1 & -0.5 & -0.3 & 0 & 0.3 & 0.5 & 1 \\
$\delta_{R}$ & 162 & 125 & 110 & 88 & 66 & 51 & 15 \\
Stk $_{R}$ & 5.4 & 4.2 & 3.7 & 2.9 & 2.2 & 1.7 & 0.5 \\
\hline
\end{tabular}

Soulsby (1983) suggested that the effective combined boundary layer, which would be the planetary boundary layer for tidal currents (Fig. 3), is a weighted mean boundary layer $\delta_{R}$ made up of a combination of $\delta_{+}$and $\delta_{-}$:

$\delta_{R}=\frac{R_{+} \delta_{+}+R_{-} \delta_{-}}{R_{+}+R_{-}}$,

which is equivalent to

$\delta_{R}=C_{2} \sqrt{C_{\mathrm{d}}}\left(\frac{U_{M} \omega-U_{m} f}{\omega^{2}-f^{2}}\right)$.

The value for $c_{2}$ was taken to be 0.075 following Soulsby (1983), who based it on observations of the measured mixed layer thickness from Pingree and Griffiths (1997). $C_{\mathrm{d}}$ is the drag coefficient, used here as a constant of $2.5 \times 10^{-3}$, although we know that this might change due to bottom roughness (e.g. Burchard et al., 2011). So the rotational Stokes number $\mathrm{Stk}_{R}$ will be as follows:

$\operatorname{Stk}_{R}=\frac{\delta_{R}}{h}$

\section{Results}

Let us assume that we are in a shelf sea of about $30 \mathrm{~m}$ depth at latitude of $54^{\circ} \mathrm{N}$, with a $\mathrm{M}_{2}$ tidal current that has a semi-major axis amplitude $1 \mathrm{~ms}^{-1}$ and an ellipticity that changes between -1 and 1 . The results in Table 1 show that the Stokes number varies between 0.5 for $\varepsilon=1$ and 5.4 for $\varepsilon=-1$, with a value of 2.9 for the rectilinear currents.

In the northwest European Shelf Sea, it will be more common to find values of the ellipticity between -0.5 and 0.5 . To test the above ideas in a realistic scenario, simulations from the operational model of the Irish Sea will be used (http://cobs.noc.ac.uk/modl/polcoms/).

POLCOMS is a three-dimensional hydrostatic baroclinic model that was designed to be particularly suited to modelling shelf sea regions. A full description including the equations may be found in Holt and James (2001). The model grid is formulated on the Arakawa B grid (Arakawa, 1972), using terrain-following $\sigma$ coordinates. It also uses a piecewise parabolic method (PPM) advection scheme (James, 1996) and a $\kappa-\varepsilon$ turbulence closure scheme from the General Ocean Turbulence Model (GOTM) as implemented by Holt and

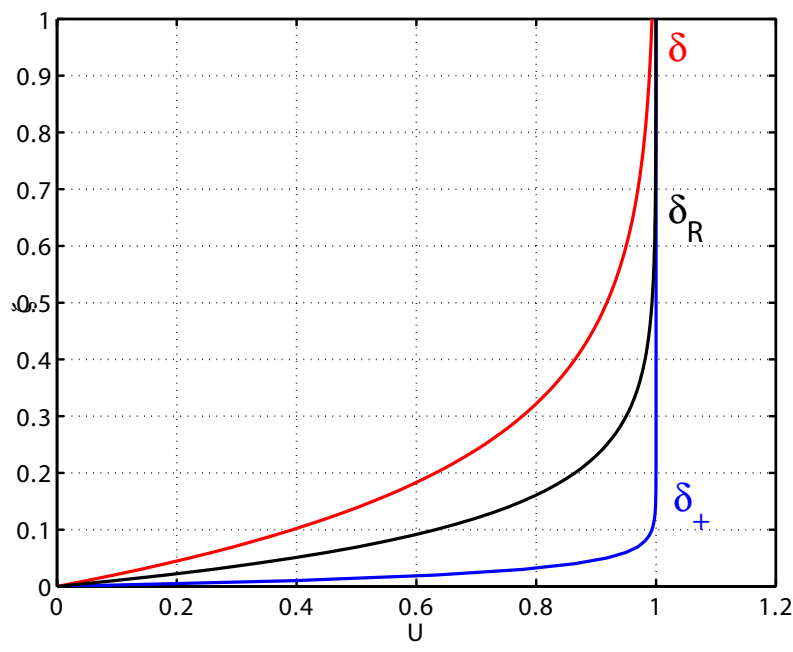

Fig. 3. Schematic of the rotational boundary layers showing the rotational boundary layers: blue cyclonic, red anticyclonic and black the resultant planetary boundary layer.

Umlauf (2008). Full details of the Irish Sea implementation can be found in Holt and Proctor (2003).

The POLCOMS Irish Sea model covers an area from $7^{\circ} \mathrm{W}$ to $2.625^{\circ} \mathrm{W}$ and $51^{\circ} \mathrm{N}$ to $56^{\circ} \mathrm{N}$. The resolution is $1 / 60^{\circ}$ latitude and $1 / 40^{\circ}$ longitude (or about $1.8 \mathrm{~km}$ ), and has 30 vertical levels; the model bathymetry is shown in Fig. 4. The data used for the analysis of tides and stratification cover the period from March to September 2007. This is so that the tidal analysis during these 6 months and the density values used for calculating stratification are an average of half of the year when the sea surface is being heated and tidal mixing fronts develop.

The ellipticity in Fig. 5 shows maximum values of 0.5 in northern Liverpool Bay, Cardigan Bay, at the centre of the deep area west of the Isle of Man and in the Celtic Sea. There is a minimum of about -0.3 in the southern Liverpool Bay and has values closer to zero elsewhere. This difference in the tidal ellipse rotation might result in a change of about $25 \mathrm{~m}$ between the rotational and non-rotational bottom boundary layer $\left(\Delta \delta=\delta_{R}-\delta\right)$. Figure 6 shows that the largest changes are in Liverpool Bay, Cardigan Bay and the Celtic Sea, where the bottom boundary layer is overpredicted at $>25 \mathrm{~m}$ (regions in red) and underpredicted by about $10 \mathrm{~m}$ (cyan areas) when using the non-rotational formulation; as expected this will be well correlated with the ellipticity.

We then calculate the Stokes number for the rotational and non-rotational frictional depth (Fig. 7); the maximum value is found when the frictional depth is the same as the water column depth. We can observe again that the non-rotational Stokes number overestimates the values in the shallow parts of the eastern Irish Sea (i.e. Liverpool Bay and Cardigan Bay) where Stk $_{R} \sim 1$. This is also apparent in the Celtic Sea and 


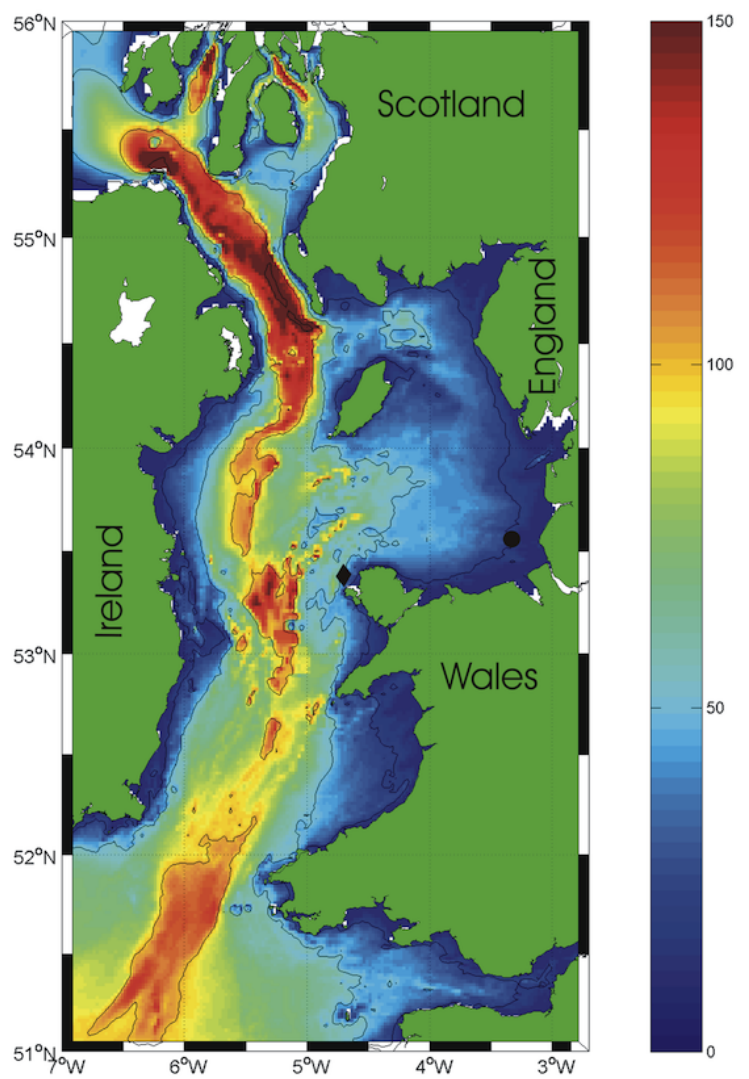

Fig. 4. Bathymetry of the Irish Sea used by POLCOMS.

in the deep area west of the Isle of Man, although here it is less critical as $\operatorname{Stk}_{R} \sim 0$.

\section{Prediction of tidal mixing fronts}

Soulsby (1983) hypothesised that the boundary layer constraint predominates, the positions of tidal mixing fronts are set by the outcropping of the tidal bottom boundary layer at the surface and that we should really take into account the earth's rotation when we define the fictional depth. If the boundary layer does not reach the surface, turbulent mixing would not be able to reach the surface thermal stratification. This hypothesis was also supported by results from Simpson and Sharples (1994) and Simpson and Tinker (2009). For Soulsby's hypothesis to hold, frontal positions will coincide with $\mathrm{Stk} \sim 1$. Furthermore, better predictions will be achieved when we use $\mathrm{Stk}_{R}$ instead of Stk. To test if this is the case, Stk and $\mathrm{Stk}_{R}$ are compared with a measure of stratification. The non-dimensional buoyancy frequency is defined as follows:

$N^{2 *}=N^{2}\left(\frac{h^{2}}{B_{\mathrm{s}}}\right)^{2 / 3}$,

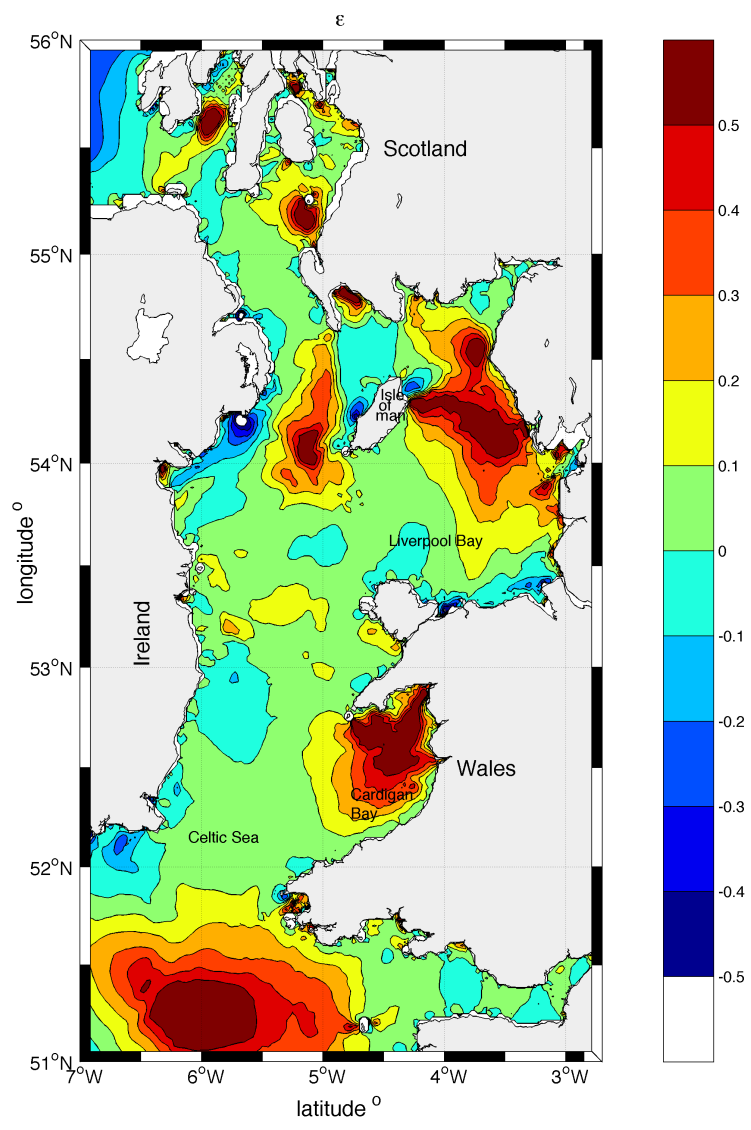

Fig. 5. Ellipticity values in the Irish Sea: positive values are cyclonic negative values are anticyclonic.

where $B_{\mathrm{S}}$ is the surface buoyancy flux due to solar heating and $N^{2}$ is the square of the buoyancy frequency. These have been calculated using the mean values for the warming half of the year (March to September).

The distribution of $N^{2 *}$ (Fig. 8) clearly shows the Irish Sea front west of the Isle of Man, the Celtic Sea front and a thermal front in Liverpool Bay. When we compare the values of the Stokes numbers with the stratification, we can observe that the Stokes number is a good predictor of the tidal mixing front position. The improvement in the predictability of the tidal mixing fronts is only obvious within Liverpool Bay due to the fact that this is a shallow area of strong cyclonic tidal currents. This is also true when the Stokes number is compared with sea surface temperature (SST) from satellite images (Fig. 9), although it should be mentioned that SST does not tell the full story about fronts and stratification as it only represents the surface signature and not any water column structure information, e.g. the strength of bottom fronts and of stratification.

It needs to be noted that for most of the frontal areas where $N^{2 *}$ values are between 750 and 850 , the tidal currents are cyclonic. Figure 10 shows the distribution of $N^{2 *}$ against Stk and $\varepsilon$. Most of the currents are cyclonic; at high Stokes 


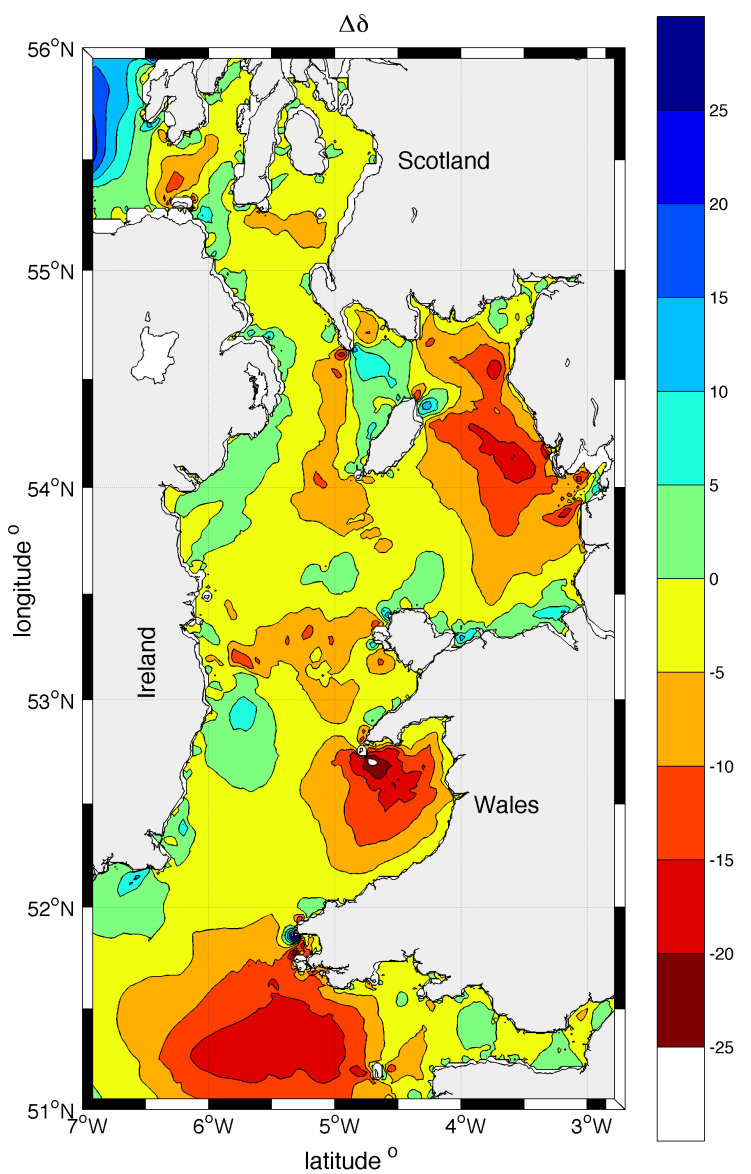

Fig. 6. Difference between the rotational-oscillating and oscillating bottom boundary layer in the Irish Sea. Negative values suggest that the boundary layer is overpredicted by the non-rotational boundary layer.
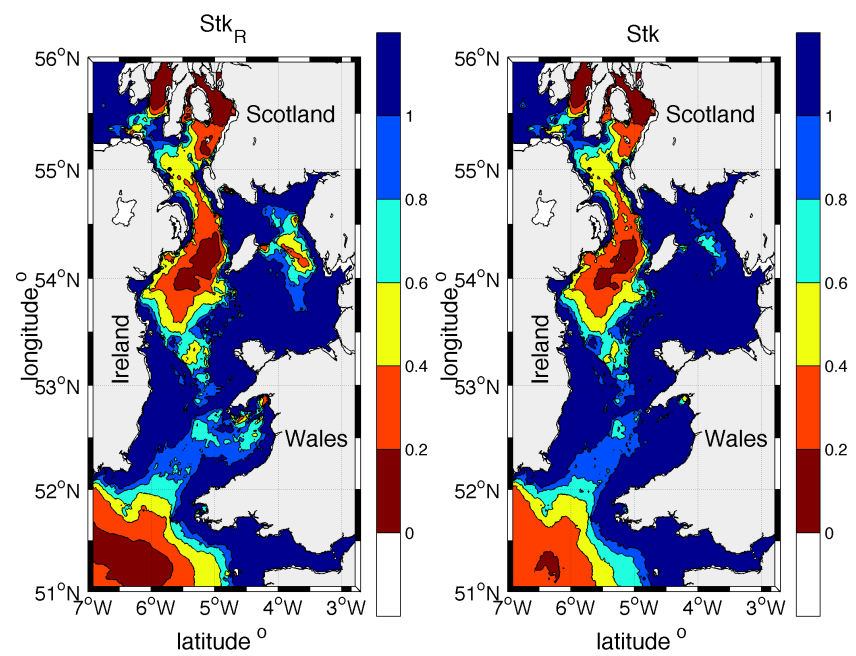

Fig. 7. Rotational (a) and non-rotational (b) Stokes number for the Irish Sea.

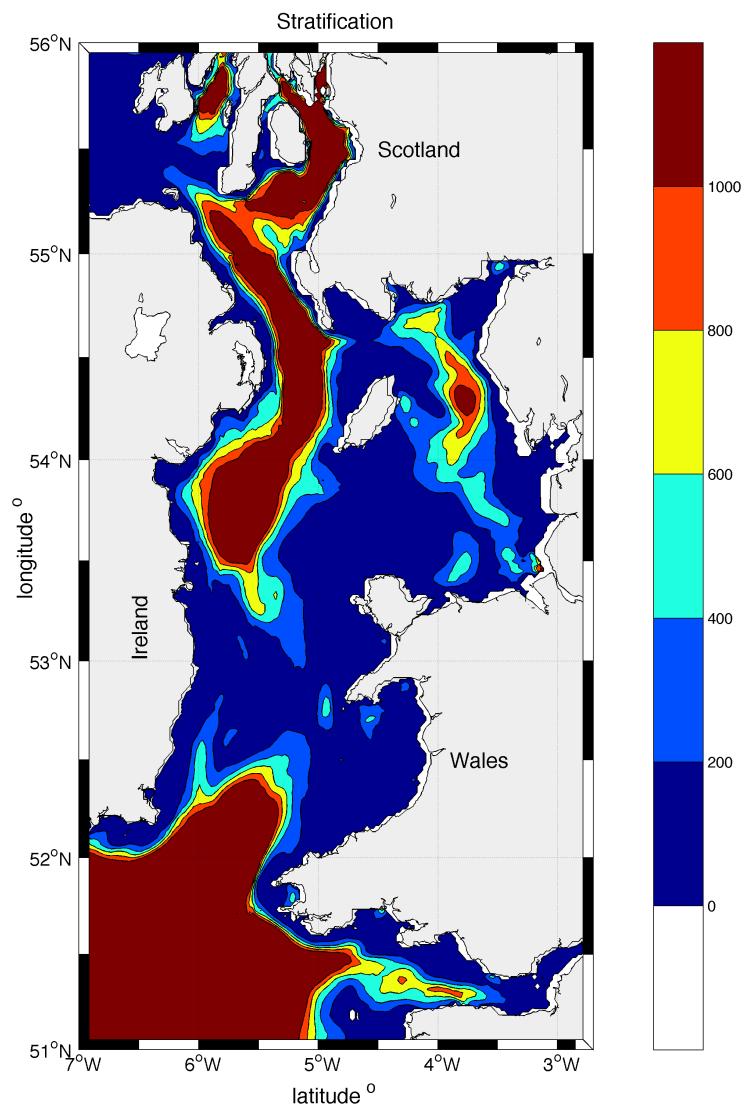

Fig. 8. Non-dimensional stratification of the Irish Sea as defined by the non-dimensional buoyancy frequency.

$($ Stk $>3)$ number the currents are almost rectilinear. Stratification $\left(N^{2 *}>1000\right)$ is possible at all positive values of $\varepsilon$; nevertheless, there is no significant stratification at ellipticity values smaller than -0.1 , and stratification also disappears at Stk $>1.5$.

\section{Conclusions}

Firstly, it is suggested that the Stokes number is the correct measure to be used concerning the ratio of the frictional depth to total depth as it is the ratio of the frictional to local accelerations.

The results presented here also suggest that the rotational Stokes number should be used to describe shallow shelf seas or estuaries, where the effect of the earth's rotation is appreciable. This could be important in places like Liverpool Bay, where the frictional layer without considering rotation can be overestimated (cyclonic rotation) or underestimated (anticyclonic rotation) by more than $10 \mathrm{~m}$. This should be considered when classifying estuaries and ROFIs (regions of freshwater influence), like those proposed by Burchard (2009) and Burchard and Hetland (2010). This is not unique to Liverpool Bay as there are other shelf seas that have shallow areas 


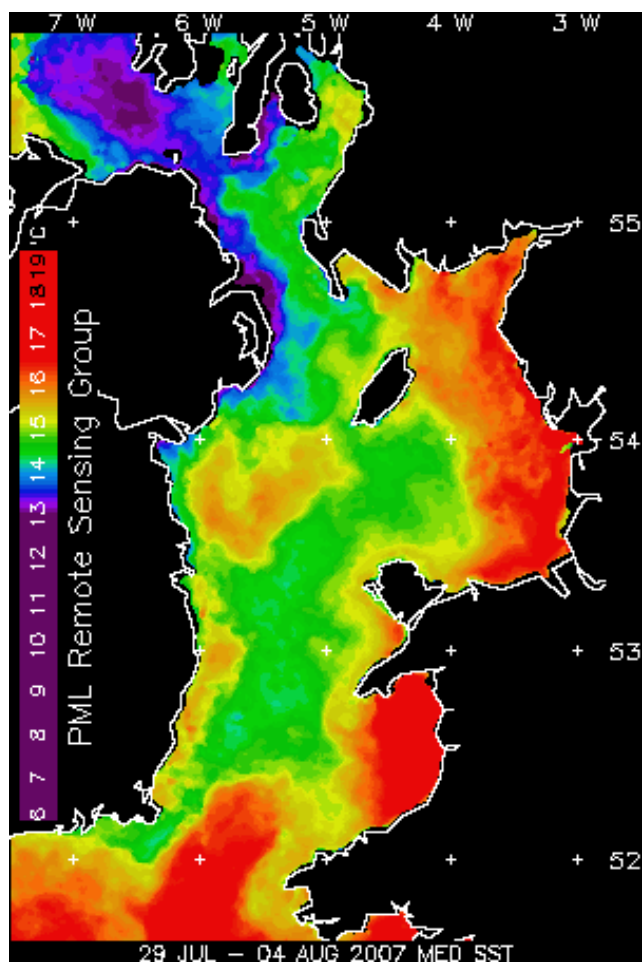

Fig. 9. Sea surface temperature weekly composite image between the 29 July and 4 August 2007. Image courtesy of NEODAAS, via the NOC Coastal Observatory.

with strong cyclonic and anticyclonic currents, e.g. the English Channel and the southern North Sea.

The Stokes number is a good predictor of the position of tidal mixing fronts, with the thermal front position occurring at values between 0.8 and 1 . The use of the rotational Stokes number appears to improve the predictions of tidal mixing front in shallow, strongly cyclonic regions. This is consistent with results found by Simpson and Tinker (2009), although here we highlight that it is even more important for shallow areas such as Liverpool Bay, in contrast with the deep areas of the Celtic Sea.

It is clear from Prandle (1982) and Soulsby (1983) that we should take into account the earth's rotation when describing the vertical distribution of tidal currents. This is evident when explaining the modification of tidal ellipses by stratification, as described by Souza and Simpson (1996) and Palmer (2011). For this process to be active, it appears to be necessary for $\mathrm{Stk}_{-}=\frac{\delta_{-}}{h} \geq 1$ so that the anticyclonic frictional layer reaches the surface; therefore, in the presence of a pycnocline the surface layer will be decoupled.

Although we have shown that the Stokes number should be used when evaluating the importance of frictional forces over local acceleration, use of the Strouhal number is still valid when assessing the importance of advection; as correctly applied by Huijts et al. (2011), this number is sometimes called the unsteadiness number.

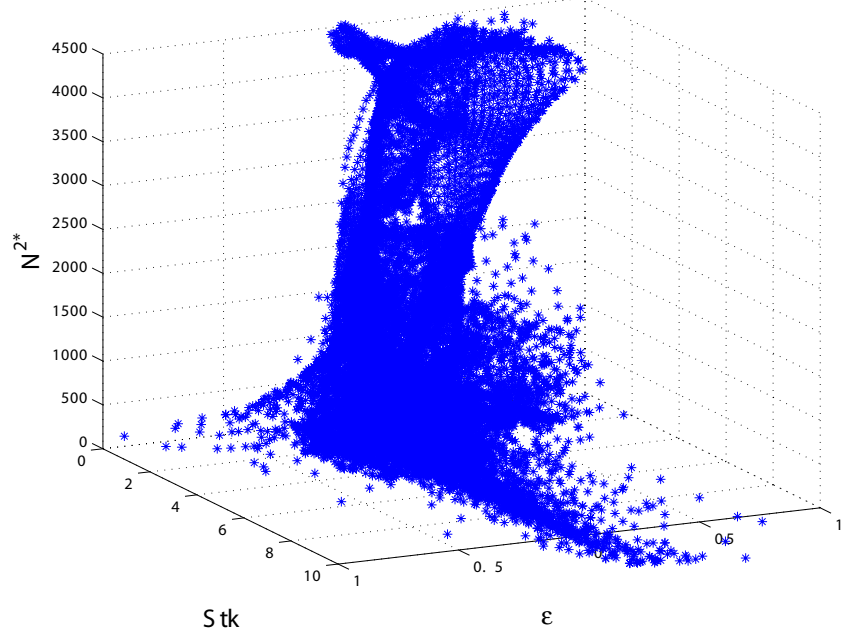

Fig. 10. Distribution of stratification $\left(N^{2 *}\right)$ against ellipticity $(\varepsilon)$ and Stokes number (Stk).

Acknowledgements. The author would like to acknowledge the National Oceanography Centre Liverpool Bay Observatory Project for the Irish Sea model data used to calculate the tidal and stratification parameters, and also for the SST satellite image through NEODAAS. He would also like to extend thanks to Prof. Arnoldo Valle-Levinson (University of Florida) for discussions on the appropriate meaning of the Strouhal and the Stokes numbers, to Jeff Polton (NOC) for pointing him to the original Stokes papers, to Parker MacCready for highlighting the Simpson and Tinker paper, and to Jenny Brown and Andrew Lane (NOC) for proof reading this article. This work was funded by NERC through NOC National Capability and Research Programme funding. The author would also like to thank David Prandle, Henk Schuttelaars and Kelly Cole for their comments that helped improve this article.

Edited by: E. J. M. Delhez

\section{References}

Arakawa, A.: Design of the UCLA general circulation model, Tech. Rep. 7, Department of Meteorology, University of California, 1972.

Baumert, H. and Radach, G.: Hysteresis of turbulent kinetic energy in nonrotational tidal flows: A model study, J. Geophys. Res., 97, 3669-3677, 1992.

Burchard, H.: Combined effects of wind, tide, and horizontal density gradients on stratification in estuaries and coastal seas, J. Phys. Oceanogr., 39, 2117-2136, 2009.

Burchard, H. and Hetland, R. D: Quantifying the Contributions of Tidal Straining and Gravitational Circulation to Residual Circulation in Periodically Stratified Tidal Estuaries, J. Phys. Oceanogr., 40, 1243-1262, 2010.

Burchard, H., Hetland, R. D., Schulz, E., and Schuttelaars, H. M.: Drivers of residual circulation in tidally energetic estuaries: Straight and irrotational estuaries with parabolic cross-section, J. Phys. Oceanogr., 41, 548-570, 2011.

Godin, G.: The Analysis of Tides, Liverpool University Press, 1972. 
Holt, J. T. and James, I. D.: An $s$ coordinate density evolving model of the northwest European continental shelf 1, model description and density structure, J. Geophys. Res., 106, 14015-14034, 2001.

Holt, J. and Proctor, R.: The Role of Advection in De-termining the Temperature Structure of the Irish Sea, J. Phys. Oceanogr., 33, 2288-2306, 2003.

Holt J. and Umlauf, L.: Modelling the tidal mixing fronts and seasonal stratification of the Northwest European continental shelf, Cont. Shelf. Res. 28, 887-903, 2008.

Huijts, K. M. H., de Swart, H. E., Schramkowski, G. P. and Schuttelaars, H. M.: Transverse structure of tidal and residual flow and sediment concentration in estuaries: Sensitivity to tidal forcing and water depth, Ocean Dynam., 61, 1067-1091, 2011.

Ianniello, J. P.: Tidally induced residual currents in estuaries of constant breadth and depth. J. Mar. Res., 35, 755-786, 1977.

James, I. D.: Advection schemes for shelf sea models, J. Mar. Syst., 8, 237-254, 2001.

Lamb, H.: Hydrodynamics, 6th Edn., Cambridge University Press, New York, USA, p. 622, 1932.

Palmer, M. R.: The modification of current ellipses by stratification in the Liverpool Bay ROFI, Ocean Dynam., 60, 219-226. doi:10.1007/s10236-009-0246-x, 2010.

Pingree, R. D. and Griffiths, D. K.: The Bottom mixed layer on the continental shelf, Estuarine Coast. Mar. Sci., 5, 399-413, 1977.

Prandle, D.: The vertical structure of tidal currents and other oscillatory flows, Cont. Shelf Res., 1, 191-207, 1982.

Prandle, D., Lane, A. and Souza, A. J.: Coastal circulation In E. Wolanski and D. McLusky, Treatise on Estuarine and Coastal Science, Elsevier, ISBN: 978-0-08-087885-0, 2011.

Simpson, J. H. and Sharples, J.: Does the Earth's rotation influence the location of the shelf sea fronts?, J. Geophys. Res., 99, 33153319, 1994.
Simpson, J. H. and Tinker, J. P.: A test of the influence of tidal stream polarity on the structure of turbulent dissipation, Cont. Shelf Res., 29, 320-332, 2009.

Simpson, J. H., Rippeth, T. P., and Campbell, A. R.:, The phase lag of turbulent dissipation in tidal flow, in Interactions Between Estuaries, Coastal Seas and Shelf Seas, edited by: Yanagi, T., Terrapub, Tokyo, 57-67, 2000.

Soulsby, R. L.: The bottom boundary layer of the shelf sea, in Physical Oceanography of Coastal and Shelf Seas, edited by: B. Johns, Elsevier, New York, USA, 189-266, 1983.

Souza, A. J. and Simpson, J. H.: The modification of tidal ellipses by stratification in the Rhine ROFI, Cont. Shelf Res., 16, 8, $997-$ 1007, 1996.

Souza, A. J., Alvarez, L. G. and. Dickey, T: Tidally induced turbulence and suspended sediment, Geophys. Res. Lett., 31, L20309, doi:10.1029/2004GL021186, 2004.

Souza, A. J., Burchard, H., Eden, C., Pattiaratchi, C., and van Haren, H.: in Coupled Coastal Wind, Wave and Current Dynamics, edited by: Mooers, C., Craig, P., and Huang, N., Cambridge University Press, Cambridge, UK, 100-123, 2013.

Stokes, G. G.: On the effect of the internal friction of fluids on the motion of pendulumns, Cambr. Phil. Trans. IX, 8; Math. and Phys. Papers, Cambridge, III, 1-141, 1851.

Strouhal, V.: Uber eine besondere Art der Tonerregung, Ann. Phys., 241, 216-251, 1878.

Thorade, H.: Probleme der Wasserwellen, Probleme Kosm Physik, 13-14, Hamburg, Germany, 1931.

Winant, C. D.: Three-dimensional tidal flow in an elongated, rotating basin, J. Phys. Oceanogr., 37, 2345-2362, 2007.

Winant, C. D.: Three-dimensional residual tidal circulation in an elongated, rotating basin, J. Phys. Oceanogr., 38, 1278-1293, 2008. 